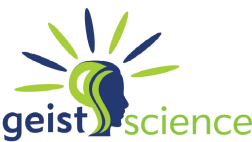

Journal of Education \& Social Sciences

ISSN: 2410-5767 (Online)

ISSN: 2414-8091 (Print)

Critical Assessment of English Examination Paper of B.Com Degree Program with Respect to Bloom's Taxonomy

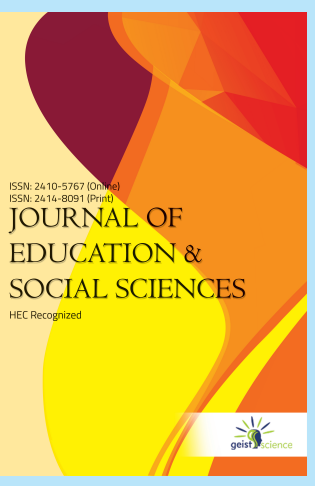

\title{
Affiliation:
}

Akhtar Ahmed Khalili

Department of English, University of Karachi, Karachi, Pakistan.

Email: colakhtarkhalili@hotmail.com

Hina H. Kazmi

Department of English, University of Karachi, Karachi, Pakistan.

Khalid M. Iraqi

Department of Public Administration,University of Karachi, Karachi,

Pakistan.

\section{Manuscript Information}

Submission Date: July 01, 2019

Acceptance Date: October 01, 2019

Publication Date: November 01, 2019

\section{Citation in APA Style:}

Iraqi, K. M. (2019). Critical Assessment of English Examination Paper of B.Com Degree Program with Respect to Bloom's Taxonomy, Journal of Education \& Social Sciences, $7(2), 1-17$.

DOI: https://doi.org/10.20547/jess0721907201 


\title{
Critical Assessment of English Examination Paper of B.Com Degree Program with Respect to Bloom's Taxonomy
}

\author{
Akhtar Ahmed Khalili * Hina H. Kazmi ${ }^{\dagger} \quad$ Khalid M. Iraqi ${ }^{\ddagger}$
}

\begin{abstract}
English is used in many parts of the world as a second or third language. The learners develop the skills of English in order to enhance opportunities for employment, higher quality education, and a good lifestyle. The purpose of this paper is to analytically review and point out the strengths and weaknesses of B.COM final term examination paper of English with special emphasis to Reading Comprehension through the framework of educational objective provided by Bloom's Taxonomy. Moreover, it provides assistance to the competitive authorities in improving their methodology of teaching of English Reading Comprehension and preparing the final term examination paper as per the framework of Bloom's Taxonomy. The sample of the study comprises of reading comprehension exercises presented to students of Undergraduate Standard Examination of Bachelors of Commerce in affiliated colleges of the University of Karachi. The questions consist of reading comprehension of the final examination from the year 2010-2016. The methodology used for the analysis is 'Content Analyses'. Furthermore, all the questions present in reading comprehensions were analyzed separately in order to test their level and underlying educational objectives according to Bloom's taxonomy. With the investigation of these comprehensions, it is revealed that how the skills of students are measured with the examination practice. Moreover, results revealed that there are various lacking and also the examiners are highly focused on the remembering skills of students which are not enough for the students of this era. Therefore, after the results in this, the suggestions are also discussed.
\end{abstract}

\section{Introduction}

Language is a mode of communication, a way of transmitting ideas and thoughts to the masses. But language is not bounded to only speech; it has various forms such as writing, drawings and signs like the ancient Egyptians used, pictorial messages, gestures, etc. a language is not limited to only verbal context, but can be developed into other contexts as per the choice of the individual using it. Man is a social animal, he has been given the intellect of choosing between rights or wrongs, the intellect to speak various forms of communicative mediums, a man has the intellect to express his emotions in the way he/she best prefers.

Language is a socially shared code, or conventional system, that represents ideas through the use of arbitrary symbols and rules that govern combinations of these symbols.

\footnotetext{
* Department of English, University of Karachi, Karachi, Pakistan. Email: colakhtarkhalili@hotmail.com

${ }^{\dagger}$ Department of English, University of Karachi, Karachi, Pakistan.

${ }^{\ddagger}$ Department of Public Administration, University of Karachi, Karachi, Pakistan. E-mail: vc@uok.edu.pk
} 
Moreover, the purpose of a language is to create a concentric circle of human activities. The human activities are now incomplete without the knowledge of languages. A language is a bridge that connects the globe to all its various parts. L. Bloom and Lahey (1978) narrated that language is a code whereby ideas about the world are represented through a conventional system of arbitrary signals for communication.

Although many novices approach statistics as yet another math subject, there are some sharp contrasts between the two disciplines (Moore \& Cobb, 2000; Cobb \& Moore, 1997). Many researchers have highlighted the importance of context in statistics: Statistics requires a different way of thinking because data are not just numbers, they are numbers with a context. Cobb and Moore (1997). Context assumes a totally different role in mathematics on the other hand.

\section{English as Global Medium of Communication}

It is the mother tongue of more than 320 million people and another 200 million use it as their second language (Baruah, 1991).

English is the world's most significant language having expansive and educative standards. Due to rich literature and vocabulary, English is used effectively in many parts of the world. The background and culture of the English language give it a very strong stabilizing effect. It is the language which is used quite commonly due to above-mentioned reasons and widely spoken throughout the world. English is a much elaborated extensive language which has not only past and present but a matter of fact it also has future as well.

In the pre and post partition times, English was considered a foreign seed of destruction for the nation and had to face a rigid criticism from the Pakistani classes. But the elite class and the schools of the elite used English or a bilingual mode of instruction whereas the government and non-elite schools preferred English only as an examination subject nothing more. After the constitution construction and awareness methods, English acquired the status of the official language. But yet is still neglected by the governmental educational policies. Certain provincial languages tried to overcome the popularity of English but failed to grasp even the minor function of its publicity.

\section{The Teaching of English Language in Pakistan}

The English language is now considered as a global medium of communication, an international bridge of unity. Due to its easy alphabetical formation and versatility of its lingual skills has given a significant value to the English language around the globe. But sadly, the department of English language in Pakistan is quite disappointing, after surveys of certain rural areas of Pakistan we have concluded that the English language in Pakistan faces difficulties because untrained teaching staff, old and ludicrous methods teaching the language, scanty resources and facilities, overcrowded classrooms, unjust and memory driven examination system and lack of exposure to good, captivating content in the textbooks. The teachers in the rural and urban areas as well teach English as 
only a subject, not as a language, this has damaged the abilities to learn the language drastically in the past decades.

Over time the English have developed into a tool of competency and intellect. The student's around the globe study the English language so they can easily communicate with the world, all major post-colonial countries and world powers use English as a mode of instruction. Pakistan has English as its 'official' language yet the faculty of English in the country is barely dragging itself. The country's major population resides in rural areas, and the standards of education in these countrysides' are shamelessly low, while the English language department is no more than a 'subject' like science, social studies, etc.

Students of the Pakistani government and non-elite schools are taught English in a rate of average ix period per week for almost ten years, but the competency of the English language in our pupils is poor, this is due to the chaotic state of teaching and the careless non-serious attitude regarding the language itself. The scholars when enter a university lack the basic skills of grammar, reading, vocabulary and even speak English.

"We hunt for shadows rather than substance. We care more for diplomas than for knowledge. It is not learning that we want, but the prize that learning brings - wealth, prestige, status, and so on. It is not knowledge that we ask for but short - cuts to knowledge, so that we can outwit the examiner".

The students are prepared only for the term or annual examination using a rather rooting system, the students memorize the context which the teacher has presented in the year-long period, and let's say copy paste it in the examinations. According to Baruah (1991) our present education system is inadequate to meet the requirement of development and use of language skills. Instead, this results in no creative skills no proper usage of grammar but simply a case of theoretically following what was direct to them. The paper pattern is a pathetic and too simple direct style which prompt the students to cram and memorize only the same answer pattern. This gives rise to the system of cramming during examinations rather than properly using language to answer the question by one owns mind.

The educational system of Pakistan has clearly failed to promote the English language as a mode of instruction. The teaching standards of the Pakistani schools are poor and saddening, the competency of teachers themselves are low and the proficiency of the students developed are not at all according to international standards. The methods used for tutoring are weak and obsolete. "Teachers teaching English to secondary students are not so competent in teaching the language. Oral competence of teachers teaching English is very poor; reading competence is found to be poor and writing competence seems to be somewhat poor" (Franklin, 1997).

\section{The Lack of Reading Comprehension Researches in Pakistan}

Pakistan is a developing country and it has been struggling poorly in the educational department for quite a long time. The educational policies and literacy rates have been neglected this is a serious problem in our governance. The public and private schools face a strict debility of educational research as well as the factor the education has become 
a commercial business in Pakistan. Upon survey of the Higher Educational Commission web library and universities around the country, we have found that the research on reading and English language learning is drastically low. Almost 300 students per year around the country master in English but none of them ever research on the subject. The English language and other teaching methodologies have become a taboo in Pakistan because no one has bothered to improve the education but have simply acquired it as a mean of income or commercializing. Any research that has been conducted regarding this topic of reading comprehension abilities is by Aga Khan University students and only one or two have properly researched on this topic.

The objectives of this study include first is to critically review and point out the strengths and weaknesses of B.COM final term examination paper of English with special emphasis to Reading Comprehension through the framework of educational objective provided by Bloom's Taxonomy. Secondly, this study provides assistance to the competitive authorities in improving their methodology of teaching of English Reading Comprehension and preparing the final term examination paper as per the framework of Bloom's Taxonomy.

The research is significant as it was observed that no other significant or helpful research was conducted in Pakistan regarding reading comprehensions and strategies that determine the strengths and weaknesses of B.COM final term examination paper of English with special emphasis to Reading Comprehension through the framework of educational objective provided by Bloom's Taxonomy. The thesis will be helpful in the development of research and in creating a new era of educational support and interest in improving reading comprehension skills in students across the country. The students and teachers are the essential categories that would get many benefits from the research as after the analysis students and teachers would be able to know the actual problems of lacking in the subject. Also, what we can do to improve our educational system. This study is helpful in giving a path to more and much-needed research based on the English language. These research will play a crucial role in the improvement of learning of English Language in Pakistan. In addition, the following paper will result in a better methodological strategy of learning and teaching English. The current research is advantageous for the students and teachers as the analysis highlights the prevailing problems in the design of examination papers and reading exercises for the students.

\section{Literature Review}

\section{Theoretical Background}

The study is established on the learning taxonomy proposed by Benjamin Bloom along with an educators committee to provide students with objectives which created a holistic and firm way of educating themselves. The framework for the research is designed on the basis of six levels suggested in the taxonomy to perform the content analysis of undergraduate English examinations i.e. Bachelors of Commerce. 


\section{Learning Taxonomies}

The word "taxonomy" means the classification of something. The learning taxonomies are the classifications of the methods of learning adopted by the masses. The most frequently asked question that is raised is what is the desired learning? According to a single approach, the educational outcomes of observable behaviors which provide milestones of achievements in learning, the behaviors do not justify the learning process but are evidence of how the learning process is taking place. The assessment then can record the achievement of learning outcomes through the recognition of these behaviors. Learning taxonomies, nonetheless, can provide an apparatus for evaluating what the teachers have addressed in their teaching. In judging the level of learner's wisdom, teachers frequently ask these questions

- Do they know it?

- Do they understand?

- Have they grasped the key concepts?

It is applicable to replicate on whether all these questions asking the same thing or are the slight differences important in reflecting upon the learning? The experimenters finally concluded two frequently used taxonomies.

- Bloom's Taxonomy by Benjamin Bloom

- SOLO (Structure of the Observed Learning Outcome) Taxonomy

In the present paper, the research is conducted under the light of Bloom's Taxonomy.

\section{Bloom's Taxonomy}

Benjamin Bloom along with an educators committee designed a taxonomy which provided students with objectives which created a holistic and firm way of educating themselves. A theoretical framework which could be used to progress communication skills among examiners (B. S. Bloom et al., 1956).

The main objective of this taxonomy is to pull together the behavior and skills of the students and then grooming their attainments to a more extensive level. Bloom's earlier taxonomy was a single approach of classification describing learning patterns and behavior representations. The learning tactics adopted by teachers and then provided to the students were to be a form of assessments to evaluate the general learning ability of a child, the questioning was also faster than just simple copy to book framework. This required keen preparation and very good command of the subject for teachers so that they could create more authentic material and assessments for the students. 


\section{Figure 1}

Formation of knowledge and skills by Bloom's taxonomy

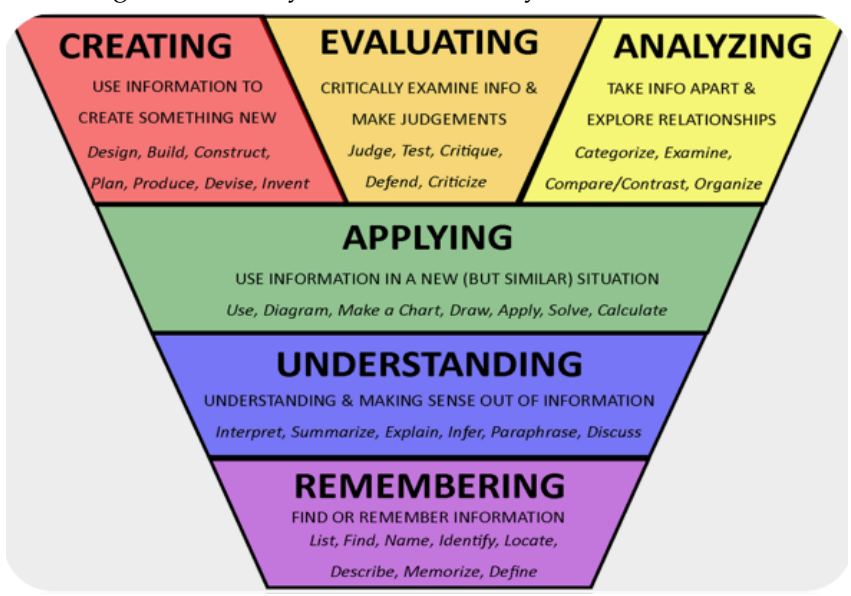

\section{Lower Thinking Skills}

The lower thinking skills are Applying, Understanding and Remembering or recalling of the content or knowledge. These skills make use of the knowledge learned in various forms. What the student has actually understood, he/she increases his/her skills in that vocabulary or problems, while lastly recalling for the long term memory or memorizing the content for further usage. These are lower skills as this is only aimed at the material of the textbook etc. no creative or mental exercises are not required. These can be easily developed by mediocre and untrained teachers.

\section{Higher Thinking Skills}

The higher thinking skills are creative, imaginative tasks to improve and develop the mind. The students do not focus on the lines of the paragraphs as to say but they learn between the lines and understand various situations of learning through the content provided. The creating of new guidelines or patterns by using the information in innovative designs, analyzing two different situations finding the similarities or difference then evaluating the knowledge by the construction of given knowledge are considered as higher skills of learning. In 2001, the Bloom's taxonomy was revised and shortened or perhaps more simplified by Anderson and Netravali (1976). They produced the ideal example for the Bloom's taxonomy, they did take out many core chains but yet the explanations of cognitive and meta-cognitive affective skills of thought and knowledge created an easier understanding of the taxonomy. 


\section{There are three spheres of influence which were identified by Bloom: -}

1. Coqnitive Development: This involves objectives relating to the development of acquired and applied leaming using various forms of reasoning, perceptions and learning plans.

2. Affective Development: This deals with the behavioral regards towards the learning process, attitudes, skills, intuitions and feelings towards learning backgrounds.

3. Manipulative or motor- skill development: This refers to the lower skills of learning, this area of development has not been much emphasized on but yet it's a significant field for students that lack or are slower the average children.

The table below is a revised version of the Bloom's taxonomy; it shows in details the four-dimensional cognitive learning process. The six categories that are expanded and structurally explained in details to how a student may achieve mastery over one category and then move on to another, this helps in the development of mental learning capacities.

Bloom's taxonomy is often considered a ladder to the higher skills of learning, which should be followed step by step. Bloom anticipated that almost all students can learn at a comparatively higher level.Higher levels of quizzical approach are the most excellent ways to reinforce the mental stamina of a learner.

1. The first level of thinking on Bloom's Taxonomy is knowledge. Bloom had defined this as a method of remembering and representing the present knowledge of the student or teacher. So the level of mental capacity could be judged properly.

2. The second standard of Bloom's Taxonomy is comprehension. This is when the student uses the given knowledge and guideline to create or groom his/her communicative and literal skills. 3. Application of the knowledge is the third level of Bloom's Taxonomy. This helps a student to apply the learned skill and test his/her 
mastery in the category, the level of application is as a testing ground for the students.

\section{Table 1}

Revised Bloom's Taxonomy

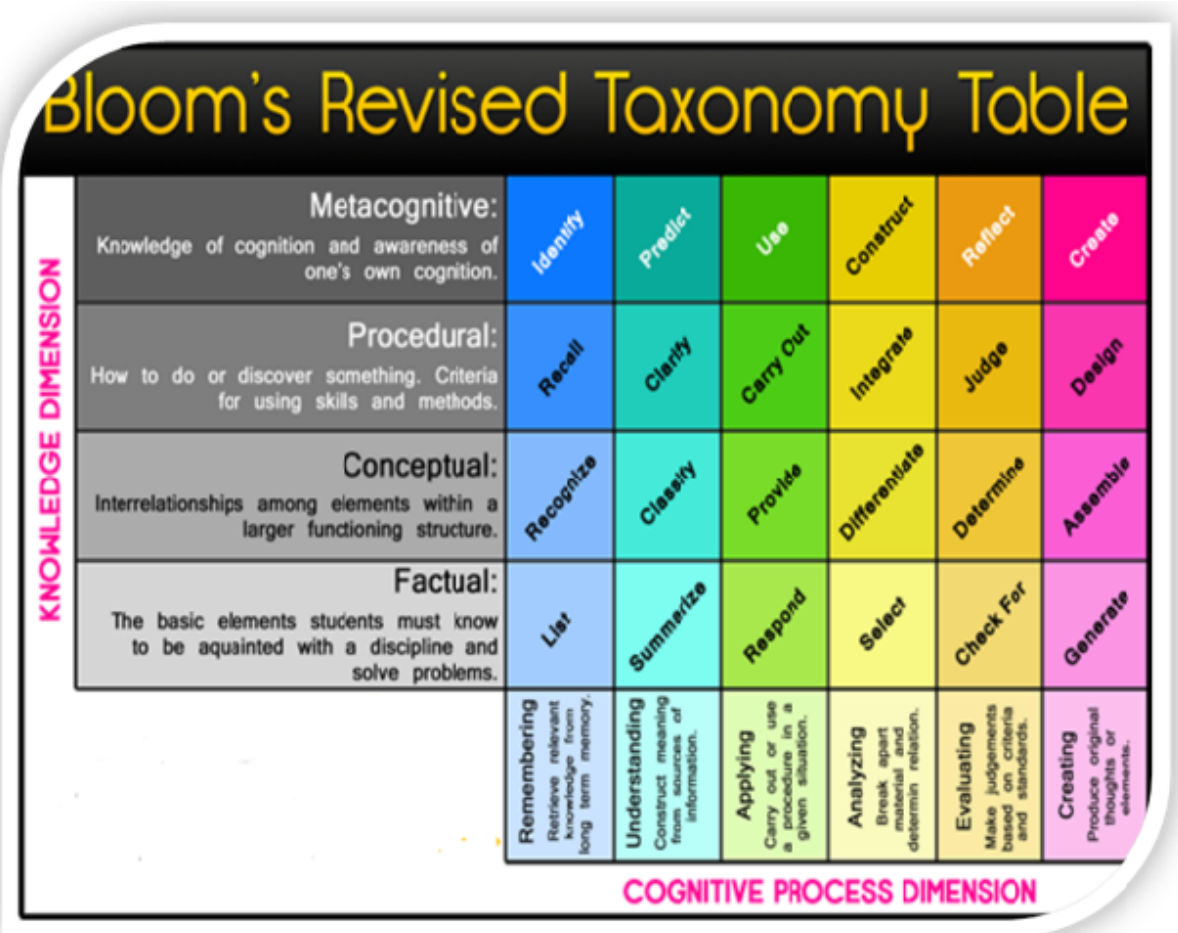

3. Application of the knowledge is the third level of Bloom's Taxonomy. This helps a student to apply the learned skill and test his/her mastery in the category, the level of application is as a testing ground for the students.

4. Analysis of the context is the fourth level. Now, the fourth stage allows the students to break down the context and research more on the context and figuratively use the information.

5. Synthesis is the fifth level of the taxonomy. Synthesis is a high level of learning the student's puzzle and recreate the context, they use all their skills and formulate it int their use and problem-solving.

6. The highest level of Bloom's Taxonomy is evaluation. "Evaluation involves making judgments about the value of the material" (B. S. Bloom et al., 1956). The highest level of evaluation occurs only when the mastery of all five levels is completed, this involves the student in creating a solution of related tasks and then reforming the skills in the meta-cognitive appraisal. 


\section{Review of Related Literature}

Agrawal (1983) conducted a research on the topic "A Study of Reading Ability in Relation to certain Cognitive and Non-cognitive factors". The objective was to observe the cognitive and non-cognitive abilities to read in school. This was conducted on 400 students of both genders equally divided in half; the students were from all classes and schooling systems. They all had some personal experience of reading comprehension tests and vocabulary building challenges. Data collection and analysis deduction methods were used to find the results. The female candidates had better test scores in academic activities and were more significant in educational progress as compared to male candidates. ANOVA results specify that the main effect of sex was significant in study habits, academic achievement, verbal and non-verbal intelligence, anxiety, neuroticism, parental attitude, and ideal self. Personal and cognitive factors were more meaningful in the context of reading ability scores than the non-cognitive factors considered in the study.

Dass (1984) conducted research "A Study of Reading Comprehension in English of Students of English Medium Secondary Schools of Standard X of Central Gujarat in the Context of Some Socio-psycho Factors". The objective was to test the reading comprehension skills in central Gujarat, among both male and female students. The research was conducted on 873 students having 384 girls and 489 boys from randomly selected schools of the city. A written exam was taken in which students had to read and understand a passage then evaluating it, recreating it and doing tasks asked. The reading comprehension and SES reading factors were divided so that each student was judged properly. The conclusion was remarkable; students with high SES scored better, and among high scorers, there were also students that had good leadership qualities. Radicalism had a good effect on the reading comprehension those with high levels scored likely more.

Dhanger (1985) did a comparison between backward classes and non-backward classes of urban and rural areas of India. This included reading abilities of students and factors like intelligence, birth orders, family status, radicalism, anxiety and schooling systems these students were being educated in. The Motibhai Patel's General Ability Test, Boxall's Test of School Anxiety (adapted), the adapted Smith's n-Ach Measure and Trivedi and Patel's Test of Reading Ability was used for collecting the data. 1811 pupils of grade $X$ of 79 secondary schools were selected for this research.

The results revealed that birth order had a significant effect on the reading comprehensions the first in the order were better at reading than those of lower order. Students with good families had better educational abilities and were likely more intelligent. Small families had a better impact on children than larger families as levels of anxiety and hypertension was considered less and more attention gave students the confidence to gain an education.

Kantawala (1980) investigated the Reading Attitudes of High School Students. The researcher provided good verbal and written patterns for reading comprehension abilities. The variables like sex, family size, and intelligence and study habits were also given priority in this study. The survey was taken in randomly selected schools of all age groups and genders. After reading tests and ranking the average reading ability the research concluded that Reading attitude was a function of grade. The scale did not indicate a sig- 
nificant relationship with cultural settings, sex, differences, age groups, and birth order. The higher the SES, the better was the reading attitude. This was true irrespective of the levels of their grades. Between reading habits and reading attitude, there was a significant positive relationship. Students of small size families had better reading comprehensions.

The role of early linguistic awareness in Children's Reading and Spelling. The research was to examine the reading abilities of people and observe their phonics and lingual skills. These are important in reading comprehension acquisitions. The research was conducted on a group of children of the age group 4 till 11 in a period of two years. The children were given knowledge and testing of phonics, syntax and evaluation of vocabulary. Different variables like intelligence, anxiety were also tested. The results showed that children had abilities to develop alphabetical codes and develop word spelling and word recognizing. The phonics helped them identify unfamiliar but similar sounding words properly.

Linek (1993) determined the relationships among Teacher Attitudes, Teacher Theoretical Orientation to Reading, Student Attitudes toward Reading, and Student Reading Achievement in Urban Elementary Grades. It was about how student teacher relation and the teachers' aptitude affect the reading orientation of the students. The study was conducted on teachers and students to get better data analysis. The research was conducted on 800 students of all sexes, in urban population and almost 70 teacher attitudes and behavior was also recorded. The reading and instruction by teachers' was also put in notice to see the achievement of the students. The result proved that the better the teacher attitude the more the student achieves, this is like moral support and inspiring factor for the students.

Taylor (1992) studied Family Support of Children's Reading Development in a Highly Literate Society. The research was performed on Icelandic families to see what the effects of family literacy on students are in the educational and reading development. The families were monitored drastically in a yearlong period and various experiments of reading and literacy were performed on them, including family activities, library usage, conversation, etc. The results were positive in this manner the family literacy did affect the students' learning and reading activities and outputs. The support of the family engaging in educational activities groomed the child's mind drastically. The leisure reading habits has effects on the student's mind. The will to read and how this will can be developed in the majority of students. She found the parental motivation and socializing with proper literate people gave inspiration to reading readiness.

\section{Methodology}

In this study, exploratory research purpose with qualitative is used as it was observed that no other significant or helpful research was conducted in Pakistan regarding reading comprehensions and strategies of Final examination of B.Com papers. Exploratory research is designed to conduct research about any problem and when there are few or no earlier studies to refer to or rely upon to predict an outcome. This study is based on a qualitative approach. In addition, a research design is "Content Analysis" as it is a technique of research that is used for making replicable and relevant interpretations on the 
basis of past events or material. Many researchers have defined content analysis in different perspectives such as Berelson (1952); Krippendorff (2018) defined it as an organized, replicable method for reducing many words of the script into fewer content types on the basis of coding explicit rules. The definition of Holsti is further elaborated by Wheelock, Bebell, and Haney (2000); Stigler (1999) that content analysis is not only limited to the area of textual analysis and thus it is also applicable to other domains for instance coding drawings of students or coding of actions witnessed in videotaped studies.

The targeted audience includes the students of B.COM. The sample size of this research includes the past papers of English of the B.COM from 2010 to 2016. The past papers were taken from the faculty of the University of Karachi. It also maintains ethics and standards in order to provide appropriate research. In this study, the data analysis is done on the basis of past papers of Bachelors of Commerce. So the content analysis is done on the basis of actual past papers and all passages was extracted from those papers. Moreover, in such type of research i.e. content analysis, there are high chances of the improper analysis of data in order to have required results. This is one of the major ethical issues as it is the reason behind stating false and confusing conclusions in research. Hence, in this study, the proper data analysis is conducted by using fair means.

\section{Data Analysis}

\section{Content Analysis of English Paper of B.COM Degree Program}

\section{Content Analysis for the Year 2016}

There are two comprehensions one is unseen and the other is seen. Seen comprehension is extracted from the textbook. In the English Paper of 2016, it is observed that in both passages there are three questions that come under the stage of Remember and other three lies at Understand stage. Whereas, the remaining three questions come under the stage of apply, create, and analyze. Hence, it shows that examiners mostly focus on remembering and understand the stage of Bloom's taxonomy.

Some questions are designed in a way that students just need to recall previously learned information and on the basis of that, they have to select one word that has to be similar to the mention meanings. Moreover, in some questions students are required to just identify the answer and then need to explain that concept in detail. Whereas, there are single questions about apply, analyze, and create. Here students are required to organize, relate, compare, contrast, examine, the question and test their knowledge. Here the students need to apply critical thinking to establish their opinions about the acquisition of sound education. It shows that students other capabilities are not being judged at a greater extent. There is a great need to examine students' ability to analyze, evaluate, and create. 


\section{Content Analysis for the Year 2015}

There are two comprehensions one is unseen and the other is seen. Seen comprehension is extracted from the textbook. In the English Paper of 2015, it is observed that in passages there are four questions that come under the stage of Remember and two lies at Understand stage. Whereas, the remaining three questions come under the stage of apply, create, and analyze. Hence, it shows that examiners mostly focus on remember and understand the stage of Bloom's taxonomy.

In an unseen passage, the first question is all about the Remembering stage so students just need to recall the prior knowledge. The second lies at the understand stage as in this students need to explain the concept of Mughals' culture. Furthermore, the other question lies at the stage of Remember because here they just need to mention the name of that culture which was given by Mughals. Another question lies at the evaluate stage and here students evaluation skills are observed as they are required to state about the advantages of pluralistic society or we can say that here they need to make judgments about their point of view. In addition, by appraising a situation, arguing, defending, judging, critiquing, supporting, or weighing in with thoughts based on the knowledge and application they've acquired by reading the passage. The fourth question lies at the third stage i.e. Apply. In this question, students need to just think about the title on the basis of their understanding. Lastly, the question lies at the Create stage as in this students are required to build a pattern from diverse elements. In addition, by putting parts of passage together also using prior knowledge they would develop a summary. Similar to the unseen comprehension same pattern can be noticed in the questions of seen comprehension. Moreover, seen comprehension is all about remembering skills as in these questions students need to recall the information they learned during lectures or from their textbook.

\section{Content Analysis for the Year 2014}

There are two comprehensions one is unseen and the other is seen. In the English Paper of 2014, it is observed that in passages there are three questions that come under the stage of Remember and two lies at Understand stage. Whereas, the remaining three questions come under the stage of apply, create, and analyze. Hence, it shows that examiners mostly focus on remember and understand the stage of Bloom's taxonomy.

In an unseen passage, the first question is all about the Remembering stage so students just need to recall the prior knowledge. The second lies at the understand stage as students are required to explain ideas and concepts. On the basis of knowledge, students need to explain in detail about the question that why does writer say that the days of unused land are over. Another question lies at the evaluate stage and here students evaluation skills are observed as they are required to justify their opinion and decision by appraising a situation, arguing, defending, judging, critiquing, supporting, or weighing in with thoughts based on the knowledge and application they've acquired by reading the passage. The fourth question lies at the third stage i.e. Apply. In this question, students need to just think about the title on the basis of their understanding. Lastly, the question lies at the Create stage as in this students are required to summarize the whole 
comprehension. Similarly, the same pattern can be noticed in the questions of seen comprehension. Moreover, seen comprehension is all about remembering skills as in these questions students need to recall the information they learned during lectures or from their textbook.

\section{Content Analysis for the Year 2013}

There are two comprehensions one is unseen and the other is seen. In the English Paper of 2013, it is observed that in passages there are three questions that come under the stage of Remember and three lies at Understand stage. Moreover, two questions are about the analytical skills of students. Whereas, the remaining two questions come under the stage of create and apply.

In an unseen passage, the first question is all about the Remembering stage so students just need to recall the prior knowledge. The second question lies at the understand stage as it requires a discussion about the object of education. The purpose of the given question is to assess the understanding skills of students with the explanation of the ideas and concepts given to them as per their understanding. Another question lies at the analyze stage and here students are required to organize, relate, compare, contrast, examine, the question and test their knowledge. Also, students need to apply critical thinking to establish their opinions. The fourth question lies at the second stage i.e. Understand as, on the basis of knowledge, students need to explain in detail about the question that why cramming should be discouraged. In addition, in the passage, it is mentioned but here students can paraphrase a point or compare and contrast information. Then there is a question about the title so it would lie at the apply stage. Lastly, the question lies at the Create stage as in this students are required to summarize the whole comprehension. Similarly, the same pattern can be noticed in the questions of seen comprehension. Moreover, seen comprehension is all about remembering skills as in these questions students need to recall the information they learned during lectures or from their textbook.

\section{Content Analysis for the Year 2012}

There are two comprehensions one is unseen and the other is seen. In the English Paper of 2012, it is observed that in passages there are three questions that come under the stage of Remember. Moreover, in 2012 there are two questions of analyze and two questions of apply. Whereas, the remaining three questions come under the stage of understand, evaluate, and create.

In an unseen passage, the first question is all about the Remembering stage so students just need to recall the prior knowledge. The second question lies at the analyze stage as students are required to organize, relate, compare, contrast, examine, the question and test their knowledge. Here the students need to apply critical thinking to establish their opinions. Third stage about the understanding skills as it needs an explanation about the opinion of the author regarding human life. Therefore, students need to answer efficiently by reading the passage also by identifying the author's opinion in various ways. The fourth question judges the evaluation skills of students and lies in the fifth level of the 
pyramid i.e. evaluate. At this level, the students are required to justify their opinion and decision by appraising a situation, arguing, defending, judging, critiquing, supporting, or weighing in with thoughts based on the knowledge and application they have learned by reading the passage. Furthermore, fourth lies at the stage of Apply because students need to think about the title on the basis of their understanding and then implement that knowledge in the form of an appropriate title. Lastly, it lies at the Create stage in this students are required to develop a summary. Similarly, the same pattern can be noticed in the questions of seen comprehension. Moreover, seen comprehension is all about remembering skills as in these questions students need to recall the information they learned during lectures or from their textbook.

\section{Content Analysis for the Year 2011}

There are two comprehensions one is unseen and the other is seen. In the English Paper of 2011, it is observed that in passages there are three questions that come under the stage of Remember and three questions are of Understand. In addition, the remaining four questions come under the stages of evaluate, analyze, apply, and create.

In an unseen passage, the first question is all about the Remembering stage so students just need to recall the prior knowledge. The second question lies at the understand stage as it requires an explanation about the attitude towards insects. Then, the third question assesses the evaluation skills of students and lies in the fifth level of the pyramid i.e. evaluate so, students are required to justify their opinion and decision. Fourth question lies at the stage of analyze as here students need to answer after analyzing different aspects. As the question is that in what ways are insects fascinating so students need to draw connections between ideas. Moreover, they are required to differentiate, organize, relate, compare, examine, or test their knowledge as this question requires sufficient knowledge in order to give an answer. Then there is Apply and students need to think about the title on the basis of their understanding and then implement that knowledge in the form of an appropriate title. Lastly, it lies at the Create stage as in this students are required to develop a summary. Similarly, the same pattern can be noticed in the questions of seen comprehension. Moreover, seen comprehension is all about remembering skills as in these questions students need to recall the information they learned during lectures or from their textbook.

\section{Content Analysis for the Year 2010}

There are two comprehensions one is unseen and the other is seen. In the English Paper of 2010, it is observed that in passages there are three questions that come under the stage of Remember. Moreover, there are two questions that come under the stage of analyze and two under the stage of apply. One lies at the second stage i.e. understand and other one lies at the stage of evaluation.

In an unseen passage, the first question is all about the Remembering stage so students just need to recall the prior knowledge. The second question lies at the stage of Apply as students are required to implement all the learned information and knowledge in real life 
scenarios. In this question, students are required to answer about the purity of the air that why air around us never been completely pure so students should answer on the basis of their information and basically they would be using information in this new situation. Then, the third question assesses the evaluation skills of students and lies in the fifth level of the pyramid i.e. evaluate so, students are required to justify their opinion and decision. The fourth question lies at the stage of analyze as here students need to answer after analyzing different aspects. Then there is Apply and students need to think about the title on the basis of their understanding and then implement that knowledge in the form of an appropriate title. Lastly, it lies at the Create stage as in this students are required to develop a summary. Similarly, the same pattern can be noticed in the questions of seen comprehension. Moreover, seen comprehension is all about remembering skills as in these questions students need to recall the information they learned during lectures or from their textbook.

\section{Conclusion}

English is used in many parts of the world as a second or third language. The learners develop the skills of English in order to enhance opportunities for employment, higher quality education, and a good lifestyle. But unfortunately, the educational system of Pakistan has clearly failed to promote the English language as a mode of instruction. Therefore, this research aimed at analyzing the level of reading comprehension questions that are available in English undergraduate examinations.

The sample of the study comprises of reading comprehension exercises presented to students of Undergraduate Standard Examination of Bachelors of Commerce in affiliated colleges of the University of Karachi to critically review and point out the strength and weaknesses of final term examination paper of English with special emphasis to Reading Comprehension through the framework of educational objective provided by Bloom's Taxonomy. In addition, it provides assistance to the competitive authorities in improving their methodology of teaching of English Reading Comprehension and preparing the final term examination paper as per the framework of Bloom's Taxonomy. The questions consist of reading comprehension of the final examination from the year 2010-2016. The methodology is used in the thesis is 'Content Analysis' as the Content analysis is a research technique used to make replicable and valid inferences by interpreting and coding textual material. Similarly, in the paper, the content analysis of the English paper of B.COM is done in order to achieve the objective of the research. In addition, the study specifically focuses on the English paper's reading comprehension section both seen passage and unseen passage with regards to Bloom's taxonomy. Furthermore, all the questions present in reading comprehensions were analyzed separately in order to test their level and underlying educational objectives according to Bloom's taxonomy.

The analysis discovered that the reading comprehensions present in the exam were targeted towards analyzing the diverse skills of students because each question varies in terms of its objective and attempts to gauge the level of students learning. The retention level of students was also checked. Moreover, throughout the years, the question related 
to the application of certain knowledge in a new scenario or situation was included in the examination to measure the student's execution of knowledge. Lastly, the content creation skills of the students were assessed with the questions of formulating a summary of the provided passages. Besides, it is observed that in the discussed undergraduate discipline i.e. of Bachelors of Commerce there is a lacking in examining evaluation and analyzing skills of the students with the questions. Because these questions require to justify a decision or provide support or critique to a particular situation and analyzing skills requires that how the students analyze the different state of affairs, how they organize, relate and contrast the given concepts. And the focus is given towards remembering and understanding skills more.

In higher education, questions are used in exams to stimulate the thinking and reasoning process among students and determines their skills through the examination process. The current research is advantageous for the students and teachers as the analysis highlights the prevailing problems in the design of examination papers and reading exercises for the students. Throughout the analysis, it is observed that the students are not given constructive skills or the ability to create percussive compositions or the ability to understand the comprehensions that weren't taught to them by their instructor. And the exam paper holds a constructive and broad range of reading and writing questions, due to this students cannot clear or barely pass English examinations. The authorities responsible for designing the examination papers must consider this and there should be a proper monitoring system and close observation of the entire examination process that whether it is matched with the studied curriculum or not. Furthermore, the analysis provides ways to the competitive authorities for devising an improved methodology for teaching Reading Comprehension with the creation of a conducive learning environment for English learning. Similarly, the results can also be used as a tool in planning and preparing of English language proficiency tests of undergraduate level with particular emphasis on reading comprehension.

Future researches should take into account the other learning taxonomies, for instance, solo taxonomy to evaluate the examination design. The study can be conducted in a more sophisticated manner with the utilization of multiple learning methodologies such as constructivist learning to investigate the current system of English language teaching in Pakistan. Also, the focus is only directed towards reading comprehension exercises, which makes the scope of the study limited to the evaluation of only the reading skills of the students. In order to make the study more profound and in-depth, future researches should incorporate the evaluation of overall questions designed to gauge the English language proficiency for the undergraduate students, instead of only focusing on reading comprehension, Likewise, the analysis is conducted on the academic year of 2010-2016. The selected sample should be increased to provide an in-depth comparison. On the other hand, in this paper only Bachelors of Commerce students were targeted so in future authors can do analysis on English Papers of other domains as well. 


\section{References}

Agrawal, V. R. (1983). A study of reading ability in relation to certain cognitive and non-cognitive factors. Asian Journal of Psychology E Education, 13(3), 41-44.

Anderson, G. L., \& Netravali, A. N. (1976). Image restoration based on a subjective criterion. IEEE Transactions on Systems, Man, and Cybernetics(12), 845-853.

Baruah, T. (1991). The english teacher's handbook. New Delhi: Sterling Publishers.

Berelson, B. (1952). Content analysis in communication research. Retrieved from https://psycnet.apa.org/record/1953-07730-000

Bloom, B. S., et al. (1956). Taxonomy of educational objectives: Cognitive domain. New York: McKay, 1, 20-24.

Bloom, L., \& Lahey, M. (1978). Language development and language disorders. Retrieved from https://eric.ed.gov/?id=ED150613

Cobb, G. W., \& Moore, D. S. (1997). Mathematics, statistics, and teaching. The American Mathematical Monthly, 104(9), 801-823.

Dass, P. (1984). A study of reading comprehension in English of students of English medium secondary schools of standard X of central Gujarat in the context of some socio psycho factors. Fourth Survey of Research in Education, 4, 629-631.

Dhanger, S. S. (1985). A comparative study of the reading ability of the BC and non BC pupils of grade $X$ in the context of their intelligence anxiety $n$ ach and certain demographic variables. Fourth Survey of Research in Education, 5, 632-640.

Franklin, K. (1997). National vocational qualifications, Scottish vocational qualifications and competence-based education and training: From De Ville to Beaumont. Journal of Vocational Education and Training, 49(4), 511-530.

Krippendorff, K. (2018). Content analysis: An introduction to its methodology. Newbury Park, CA: Sage.

Linek, W. M. (1993). Relationships among teacher attitudes, teacher theoretical orientation to reading, student attitudes toward reading, and student reading achievement in urban elementary grades. Dissertation Abstracts International, 53(4).

Moore, D. S., \& Cobb, G. W. (2000). Statistics and mathematics: Tension and cooperation. The American Mathematical Monthly, 107(7), 615-630.

Stigler, J. W. (1999). The TIMSS videotape classroom study: Methods and findings from an exploratory research paoject on eighth-grade mathematics instruction in Germany, Japan, and the United States. DIANE Publishing: USA.

Taylor, R. L. (1992). Family support of children's reading development in a highly literate society (Unpublished doctoral dissertation). School of Education. University of MissouriKansas City.

Wheelock, A., Bebell, D. J., \& Haney, W. (2000). What can student drawings tell us about high-stakes testing in Massachusetts. Teachers College Record, 2. 